\title{
Treatment of a giant complicated distal posterior inferior cerebellar artery aneurysm: A case report and literature review
}

\author{
$\mathrm{JUN}_{\text {ZHU}}{ }^{*}, \mathrm{LIN} \mathrm{YIN}^{2 *}$, YANJUN CHE$^{1}, \mathrm{ZHAO} \mathrm{LIU}^{1}, \mathrm{XIN}^{1}{ }^{1}$, \\ $\mathrm{KE} \mathrm{ZHOU}^{1}$, BAO ZHENG ${ }^{1}$, ENYU PAN ${ }^{1}$ and JUNHUI CHEN ${ }^{3,4}$ \\ ${ }^{1}$ Department of Neurosurgery, Jingjiang People's Hospital, Jingjiang, Jiangsu 214500; Departments of ${ }^{2}$ Medical Imaging and \\ ${ }^{3}$ Neurosurgery, Wuxi Clinical College of Anhui Medical University, 904th Hospital of Joint Logistic Support Force of PLA, \\ Wuxi, Jiangsu 214044; ${ }^{4}$ Department of Neurosurgery, Renmin Hospital of Wuhan University, \\ Wuhan, Hubei 430060, P.R. China
}

Received July 31, 2019; Accepted April 17, 2020

DOI: $10.3892 /$ etm.2020.8724

\begin{abstract}
Giant intracranial aneurysms, especially giant aneurysms of the distal posterior inferior cerebellar artery (PICA), remain the most difficult and challenging cerebrovascular lesions for neurosurgeons to treat. The morbidity and mortality rates of microsurgical clipping are relatively high, and endovascular embolization is also associated with many complications. In the present report, the case of a 46-year-old female patient who presented with headache and dizziness for 3 years, which was aggravated and combined with limb weakness for 1 day, is presented. A CT scan showed a lesion occupying the fourth ventricle, with slight bleeding. A MR scan also revealed a lesion occupying the fourth ventricle and compressing the brainstem, and there was distortion of the cisterns around the brainstem. CT angiography examination showed a giant irregular aneurysm located in the PICA. After evaluation, the PICA aneurysm was removed, and the PICA was clipped via a microsurgical technique without ischemia or neurological sequelae. Long-term follow-up demonstrated that the symptoms of headache and dizziness disappeared without relapse. Based on a review of the literature, this method may represent an alternative strategy for the treatment of giant PICA aneurysms, especially for aneurysms not suitable for direct clipping or endovascular embolization.
\end{abstract}

Correspondence to: Dr Enyu Pan, Department of Neurosurgery, Jingjiang People's Hospital, 19 Nanhuan Road, Jingjiang, Jiangsu 214500, P.R. China

E-mail: jjzj0510@163.com

Dr Junhui Chen, Department of Neurosurgery, Renmin Hospital of Wuhan University, 9 Zhangzhidong Road, Wuchang, Wuhan, Hubei 430060, P.R. China

E-mail: chenjunhui101@163.com

${ }^{*}$ Contributed equally

Key words: giant aneurysm, posterior inferior cerebellar artery, clipping

\section{Introduction}

A cerebral aneurysm is an abnormal enlargement of a cerebral artery, and cerebral aneurysms may lead to poor outcomes if the aneurysm ruptures (1). Giant aneurysms are defined by the fact that their largest diameter is $>25 \mathrm{~mm}$, and these types of aneurysms represent $3-5 \%$ of all cerebral aneurysms $(2,3)$. Additionally, $15 \%$ of giant aneurysms originate in the internal carotid artery (4). The incidence of posterior inferior cerebellar artery (PICA) aneurysms is $<0.5-3 \%$ of all intracranial aneurysms, and only a few cases have been reported in previous studies (5-8). Giant complicated distal PICA aneurysms are relatively rare, constituting $<30 \%$ of all PICA aneurysms $(5,9)$. The treatment of giant PICA aneurysms is a challenging task for neurovascular surgeons and neurosurgeons, especially for aneurysms arising from any segment of the distal PICA. Surgical clipping may result in numerous complications because of the intimate anatomical relationships between the proximal PICA and the brainstem as well as the lower cranial nerves $(10,11)$. An increasing number of studies have reported that endovascular treatment is being used as both a primary or alternative method, but this is often associated with a high rate of complications such as aneurysm regrowth and brain ischemia $(7,10,11)$. Additionally, important limitations of this method are the substantial costs of treatment and the ischemic symptoms.

The present study reports the case of a giant complicated distal PICA aneurysm treated with surgical clipping and removal of most of the aneurysm. A review of the literature is also presented, as well as the discussion of topics related to the correct diagnosis and treatment of this condition.

\section{Case report}

A 46-year-old woman presented to a regional hospital after suffering from headaches and dizziness for 3 years, these symptoms were aggravated and combined with limb weakness for 1 day without any obvious cause. There was no previous history of trauma. The past medical history of the patient demonstrated hypertension and a history of hysteroscopic myomectomy. A CT scan showed a lesion occupying the fourth 
ventricle, with slight bleeding (Fig. 1). The regional hospital doctors considered that surgery would be difficult, and that the diagnosis was difficult to confirm; therefore, the patient was admitted to Wuxi Clinical College of Anhui Medical University, 904th Hospital of Joint Logistic Support Force of PLA (Wuxi, China) after 2 days. Before the patient was discharged from the regional hospital, a MR scan also revealed a lesion occupying the fourth ventricle and compressing the brainstem, and distortion of the cisterns around the brainstem (Fig. 2). A neurological examination performed upon admission revealed no other abnormalities, except for the fact that there was weakness in all limbs [Glasgow Coma Scale (GCS), 15] (4). Next, a CT angiography (CTA) examination was performed on the patient, which showed a giant irregular aneurysm located in the PICA, with a size of $\sim 3.5 \times 2.2 \times 1.8 \mathrm{~cm}$; the aneurysm was serpentiform and irregular, so it was considered difficult to clip (Fig. 3). Although simply occluding the PICA via endovascular embolization would be easy, it would not relieve the mass effect, and it may aggravate the symptoms. After discussion, the neurosurgeons and vascular interventional physicians agreed on the microsurgical clipping of the aneurysm and resection of most of the aneurysm.

After all preparation procedures were completed, a median suboccipital approach was selected to expose the aneurysm (Fig. 4). First, the foramen magnum was exposed and opened completely. Then, the dura mater was cut, and the PICA was observed (Fig. 5A and B). Subsequently, dissection was carried out along the PICA, and the basilar part of the aneurysm was explored; the PICA was then temporarily occluded to adequately expose the aneurysm and its boundary (Fig. 5C and D). It was difficult to perform clipping alone, as the aneurysm was very large. However, it was also very important to resect the giant aneurysm to effectively avoid obstruction of cerebrospinal fluid reflux. The PICA was then occluded and the aneurysm was removed with another optimal method (Fig. 5E). The aneurysm was very regular and ball-shaped and consisted of organized granulation tissue and fibrous tissues. After the aneurysm was mostly removed, brainstem compression was alleviated, and the cerebrospinal fluid moved more fluently (Fig. 5F).

After the operation, blood pressure was maintained below the typical level of $\sim 10 \mathrm{mmHg}$ systolic blood pressure. The patient left the Neurosurgery Intensive Care Unit after 3 days, with a GCS score of $15 / 15$, and without nerve dysfunction. The CT, MR, and CTA results showed that the PICA aneurysm had been clipped completely and that brainstem compression had been alleviated without complications (Fig. 6). A postoperative histopathological examination confirmed a giant irregular aneurysm and fibrous tissue proliferation (Fig. 7). A total of 6 months after the operation, the patient had a good outcome with no neurological dysfunction, and the symptoms of headache and dizziness had disappeared completely.

\section{Discussion}

The PICA is the largest branch of the vertebral artery, and ruptured PICA aneurysms can lead to cerebral infarction and cranial nerve compression in patients; some patients with severe rupture may die due to cardiopulmonary arrest $(5,12)$. Aneurysms of the vertebral artery (VA)-PICA represent only
$0.5-3 \%$ of all aneurysms and only $20 \%$ of those in the posterior fossa (12). Distal PICA aneurysms are rare, accounting for approximately one-third of PICA aneurysms $(5,9)$.

CT and MR imaging only indicate subarachnoid or intraventricular hemorrhage and/or infarction but cannot confirm a diagnosis of an aneurysm. In the present study, CT indicated a mass effect and brain edema, calcification and hemorrhage. MR imaging demonstrated a well-defined mass, with intra-aneurysmal thrombosis, a boundary, brainstem compression and no contrast-enhancing components. This information offered by MR was very important for microsurgical clipping. Cerebral angiography is the most important diagnostic modality and is the 'gold standard' for revealing the true nature of a lesion (13). In the present report, a diagnosis was confirmed using 3D-CTA, which showed a shape of serpentine aneurysm or fusiform aneurysm. However, the size of the aneurysm according to CTA was significantly different from the actual size. The reason for this outcome was the presence of intra-aneurysmal thrombosis and a thick aneurysm wall. However, an incorrect evaluation before surgery may lead to incorrect management strategies and negative consequences.

There are several management strategies for distal PICA aneurysms, such as surgical clipping, bypass, parent artery sacrifice (surgical or endovascular) and endovascular aneurysm embolization. However, different strategies have various advantages and disadvantages. Previous studies have suggested that surgical methods, including proximal clipping, trapping, and wrapping or resection, are very important and effective methods $(8,14,15)$. A prior clinical study reported that of 52 patients (11 male patients, 41 female patients) treated surgically for VA-PICA aneurysms, 47 (79.8\%) showed a good outcome, and only one patient (1.9\%) died due to cardiomyopathy during the follow-up period (10). Horowitz et al (16) also reported that 24/27 patients (88.9\%) had a good outcome and that only two patients (7.4\%) died at the 1-year follow-up. Wu et al (17) described a similarly favorable outcome rate in a single Chinese center. However, surgery may lead to a high risk of postoperative neurological morbidity because of the close relationship between the PICA and the brainstem, as well as the lower cranial nerves $(10,13,16)$.

Endovascular aneurysm embolization is very important and is the most common management strategy for PICA aneurysms. Endovascular technology and intervention materials are ideal methods, and various endovascular methods described for the treatment of PICA aneurysms include silicon or latex balloon occlusion of the aneurysm $(7,18)$. This treatment easily and effectively avoids rebleeding, cranial nerve dysfunction and postoperative complications (18). However, few articles have been published describing the long-term follow-up outcomes of VA-PICA aneurysms treated with embolization $(10,18)$. A clinical study, which included 23 patients treated with endovascular embolization of ruptured PICA aneurysms, found that the procedure-related morbidity rate was $13 \%$, with no procedure-related instances of death; a good outcome was reported in $86.4 \%$ patients and three patients (13\%) died within 2 months of treatment (18). Ogilvy et al (19) also reported that endovascular coiling of posterior circulation aneurysms, including PICA aneurysms in eight patients, resulted in a good outcome in $77.2 \%$ patients. Few publications have documented the specific recurrence rate with a long-term 


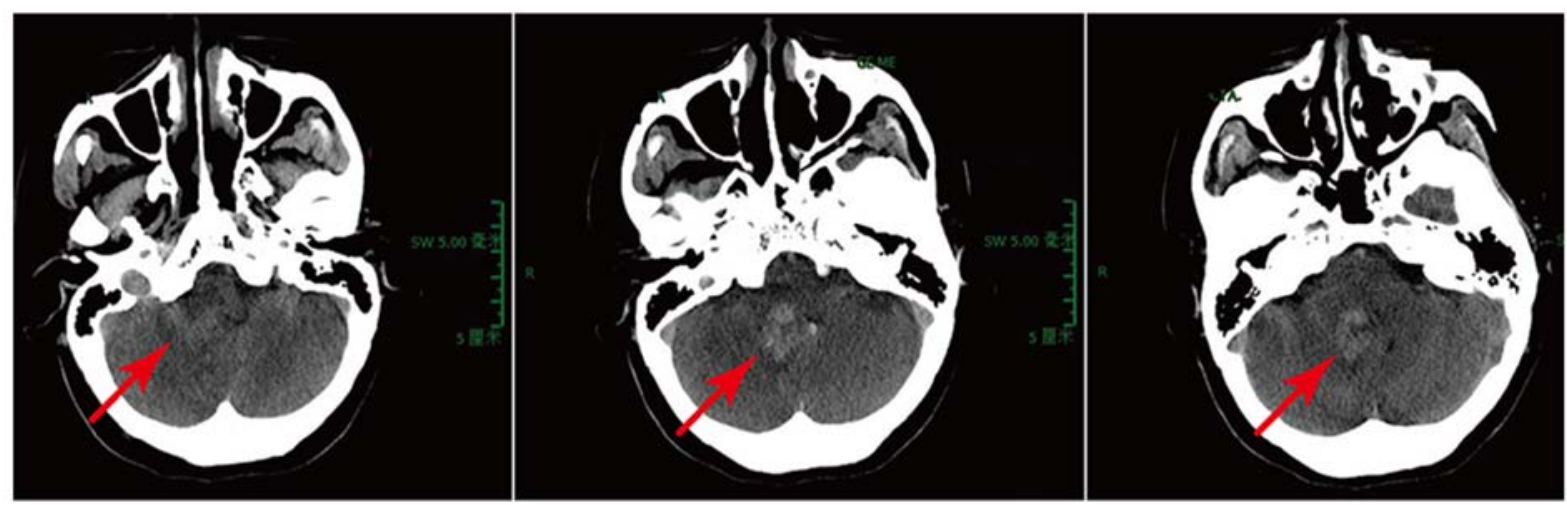

Figure 1. CT scan shows a lesion occupying the fourth ventricle with subarachnoid or intraventricular hemorrhage, and the brainstem is compressed. Red arrow indicates the lesion.

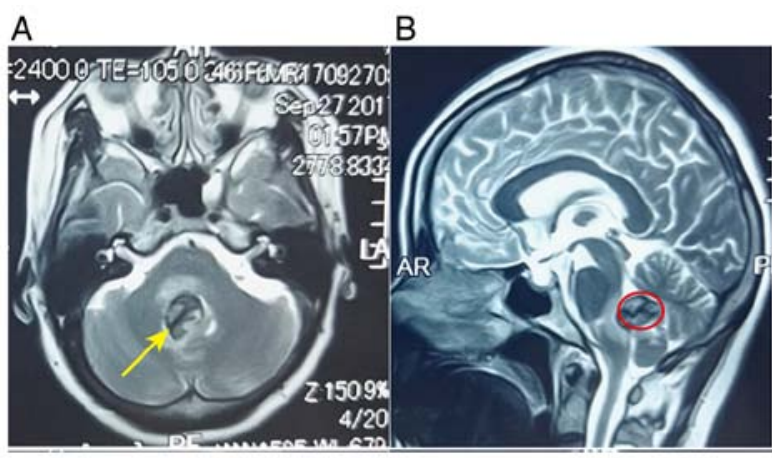

Figure 2. MR scan at the first visit to the hospital. (A) MR axial films show the boundary of the aneurysm and that the aneurysm has intra-aneurysmal thrombosis, indicated by the yellow arrow. (B) MR sagittal films show a well-defined mass, indicated by the red circle, brainstem compression injury and brainstem edema.

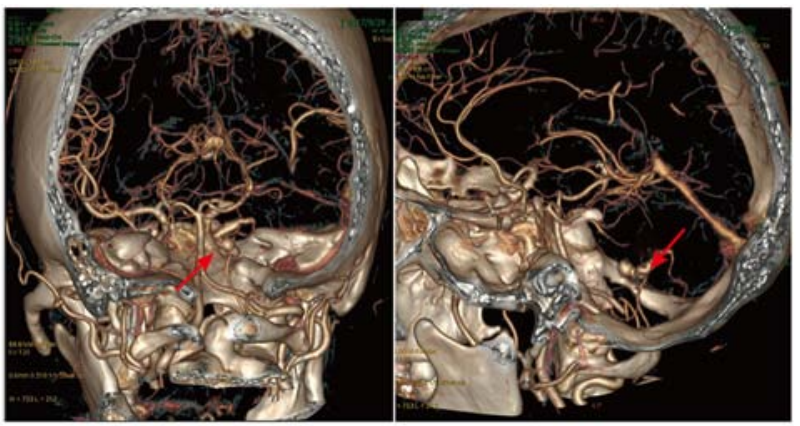

Figure 3. 3D-CT angiography scan shows a serpentine aneurysm or fusiform aneurysm, and its size is markedly smaller than the actual size of the distal posterior inferior cerebellar artery. Red arrow indicates the aneurysm.

follow-up. Byrne et al (20) reported that recurrent filling occurred in $14.7 \%$ of 259 embolization aneurysms; however, this study included only 16 PICA aneurysms.

Surgical or endovascular trapping of the dissected arterial segment, especially for the first three segments of the PICA (anterior medullary, lateral medullary, and tonsillar medullary), may lead to two main complications: An infarct in the distal territory of the PICA, and an ischemic lesion of the medulla due to the occlusion of perforators in the trapped

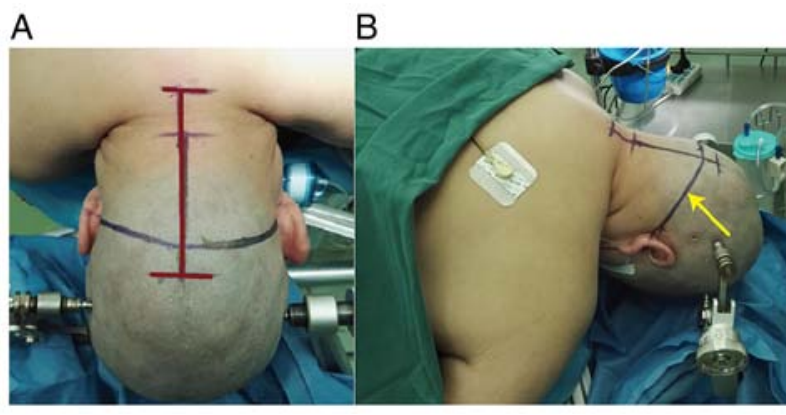

Figure 4. Surgical incision design and operative position. (A) Surgical incision; the red line indicates the head surgical incision. (B) Operative position; the yellow arrow indicates the transverse sinus.

segment (13). Intraoperative fluorescence angiography and Doppler ultrasonography are useful for reducing the rate of infarct and confirming the location of the aneurysm $(3,4)$.

The treatment time used for patients with VA-PICA aneurysms remains controversial $(11,17,21)$. Most authors have recommended early surgery (first $24 \mathrm{~h}$ ) after diagnosis, especially for Hunt and Hess grade IV and V patients $(4,11,21)$. In our center, it is recommended that ultra-early surgery (12 h) or emergency surgery may be beneficial for patients, especially for patients with severe subarachnoid hemorrhage (SAH). However, Wu et al (17) reported delayed surgery improved outcomes of proximal PICA aneurysms in a single center study, that all the surgeries of proximal PICA aneurysms were performed in a delayed fashion, several days to weeks after the presentation of SAH. No systematic clinical studies have analyzed or summarized the different outcomes of early surgery and delayed surgery. The biggest risk for cerebral aneurysms is rupture, and there is a high cerebral rupture risk with delayed timing of surgical treatment (4); hence our recommendation of early or emergency surgery. However, the disadvantage of early or emergency surgery is that this could lead to inadequate perioperative preparation for a relatively difficult operation, particularly in the case of SAH.

An understanding of PICA anatomy is essential before surgery. Lower cranial nerve palsy and cerebellar infarction are common postoperative complications that occur after VA-PICA aneurysm surgery $(10,17)$. Most distal PICA 
A

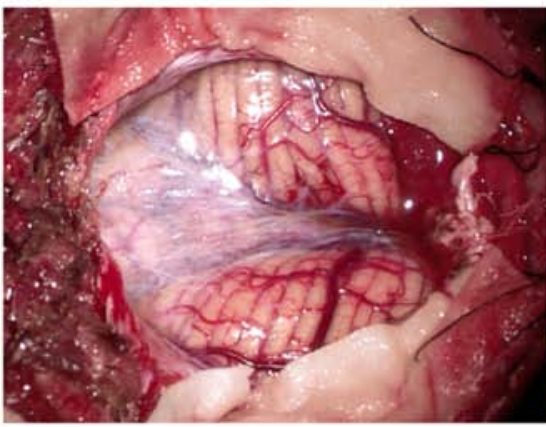

B

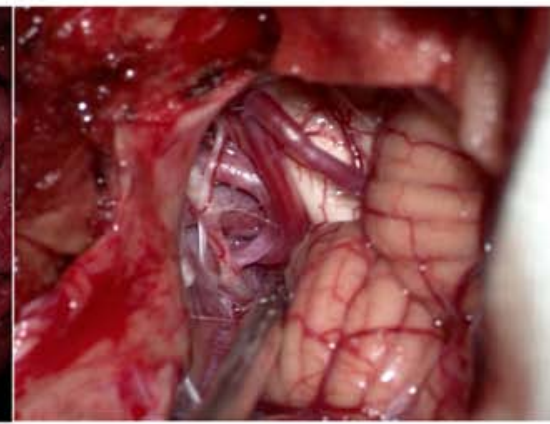

C

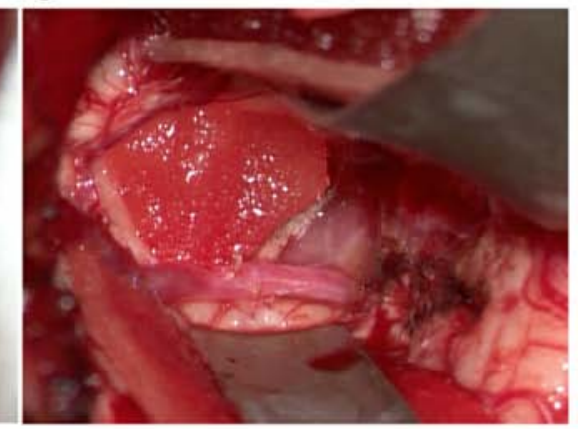

D

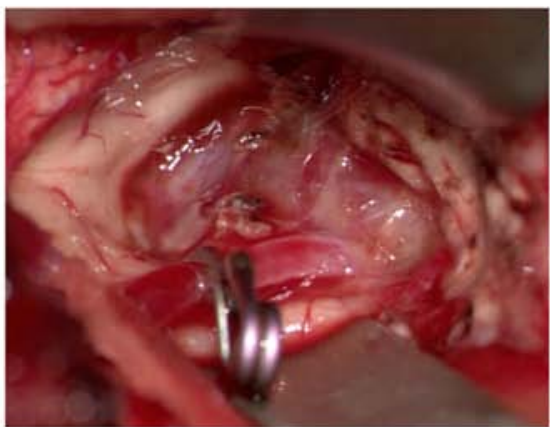

E

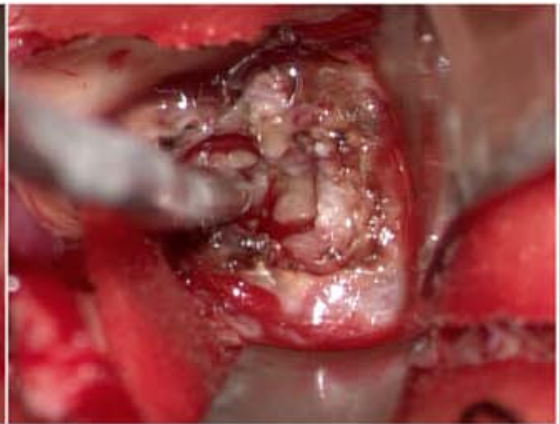

F

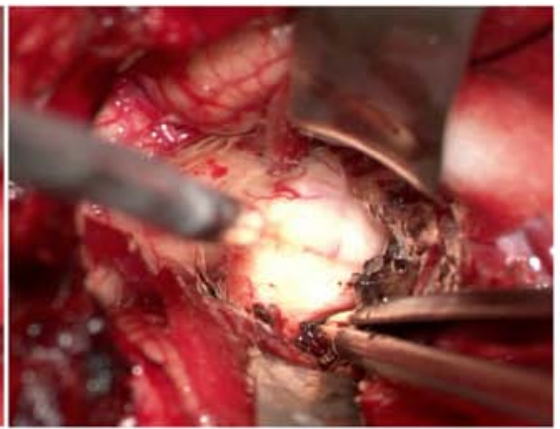

Figure 5. Surgical procedure. (A and B) Exposure and dissection of the dura and arachnoid reveals the PICA initial segment. (C) Giant aneurysm in the PICA. (D) Occlusion of blood flow to the PICA with an aneurysm clip and isolation of the aneurysm. (E) Resection of the aneurysm in a piecemeal fashion, as the aneurysm was too large to isolate and clip completely. (F) Removal of the PICA aneurysm resulted in the fluent movement of cerebrospinal fluid circulation. PICA, posterior inferior cerebellar artery.

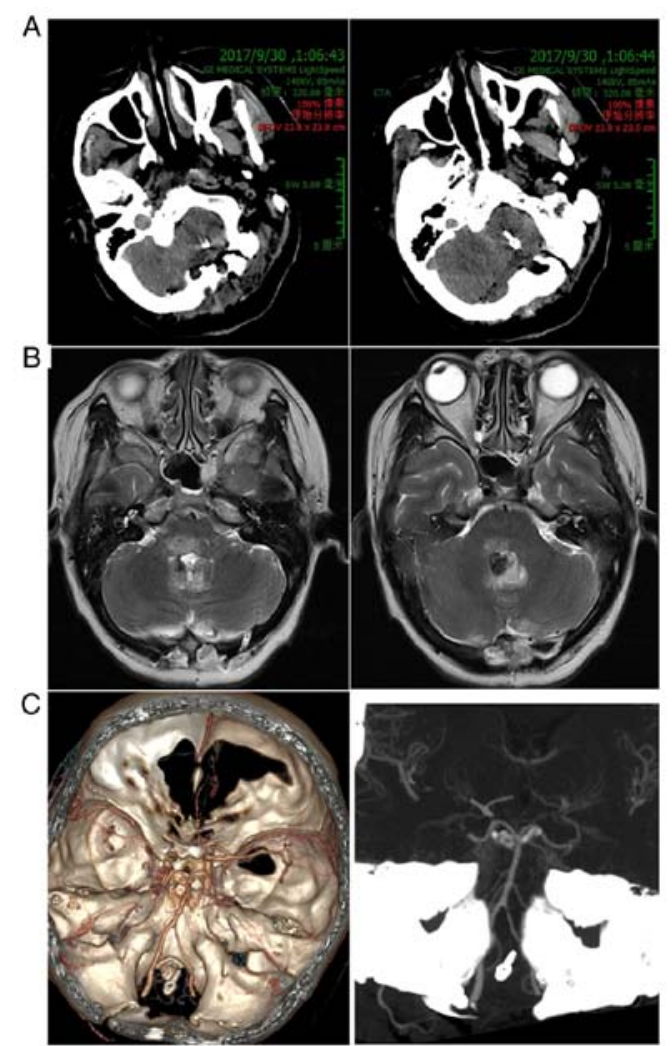

Figure 6. Postoperative examination. (A) CT and (B) MR show that the lesion occupying the fourth ventricle has disappeared, and that the brainstem compression has markedly improved. (C) CT angiography scan showed that the posterior inferior cerebellar artery has been clipped completely, and that the aneurysm has disappeared.

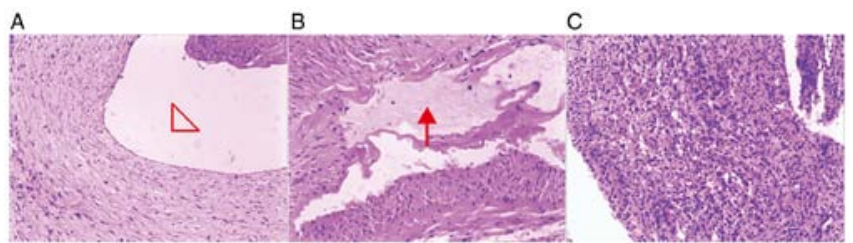

Figure 7. Postoperative histopathological examination. (A) Angiogenesis and irregular, dilated arteries, indicated by the triangle, with thickening of the arterial wall. (B) Red arrow indicates the proliferation of fibrous tissue. (C) Numerous chronic inflammatory cells have infiltrated into the wall and there is tissue clearance. Magnification, $\mathrm{x} 50$.

aneurysms are located superficial to the lower cranial nerves $(5,17)$. If the PICA is occluded, adverse effects, such as headache, vomiting, ipsilateral cerebellar ataxia, difficulty swallowing, hoarseness and dizziness, may occur, and severe cerebellar infarction may lead to obstructive hydrocephalus (17). A study by Horowitz et al (16) found that $47 \%$ of posteroinferior cerebellar artery aneurysms patients had cranial nerve IX and X palsy after operation, and only four patients (22\%) continued to have dysphagia at the 1-year follow-up. Hudgins et al (5) also reported a lower cranial nerve dysfunction rate of $23.8 \%$ compared with with Horowitz's study. Therefore, it is very important to understand the relationship between the origin of the PICA and lower cranial nerves. However, this anatomic relationship is irregular and highly variable (17,22). Al-khayat (10) described some reasons for post-operative complications in the cranial nerves after PICA aneurysm surgery as follows: i) There is a 
rapid or abrupt change caused by stretching and manipulation, as occurs in vascular surgery; ii) temporary clips may physically limit the already confined space in the posterior fossa, leading to an increased risk of mechanical trauma to the lower cranial nerves; and iii) total occlusion may cause the nerves to be more vulnerable to trauma by decreasing the blood supply. Most patients with lower cranial nerve palsy and cerebellar infarction recover completely within 6 months $(10,16)$. Intraoperative electrophysiological monitoring is useful and may avoid accidental injury during vascular surgery.

Fusiform and wide neck aneurysms present a challenge to endovascular surgeons, with a high risk of parent artery embolization, neurologic deficits and residual aneurysms $(18,19)$. More importantly, as endovascular therapeutic techniques cannot improve the symptoms of mass effect, and as microsurgical clipping may result in numerous clip-related complications, it may be necessary to utilize other methods. In the future, bypass and new flow diversion stents may become very important techniques for aneurysm treatment. Several studies have reported that PICA-to-PICA bypass or occipital artery-to-PICA bypass are able to treat distal PICA aneurysms $(11,23,24)$. However, additional studies and randomized clinical controlled trials are needed to confirm these findings.

In conclusion, giant distal PICA aneurysms are very rare and are often combined with posterior fossa syndrome. In the present study, giant distal PICA aneurysm treatment by surgical clipping and removal of the aneurysm was reported with a good outcome and without any complications. It was concluded that surgical treatment was a useful and safe option. Surgery may prevent rebleeding and can also remove space-occupying lesions, especially thrombosed aneurysms. In the future, bypass and flow diversion stent techniques may be used in the treatment of VA-PICA aneurysms.

\section{Acknowledgements}

Not applicable.

\section{Funding}

No funding was received.

\section{Availability of data and materials}

Not applicable.

\section{Authors' contributions}

JZ, LY, YC, ZL, XQ, KZ and BZ analyzed and interpreted the patient data. EP and JC performed the operation, data analysis, follow-up and revised the manuscript. JZ, LY and JC performed the histological examination of the aneurysm, and were major contributors in writing the manuscript. All authors read and approved the final manuscript.

\section{Ethics approval and consent to participate}

The study protocol was approved by the Anhui Medical University-Affiliated Wuxi Clinical College Clinical Research
Ethics Committee. Written informed consent was obtained from the patient.

\section{Patient consent for publication}

The patient provided written consent for the publication of their data and images.

\section{Competing interests}

The authors declare that they have no competing interests.

\section{References}

1. Wiebers DO, Whisnant JP, Huston J III, Meissner I, Brown RD Jr, Piepgras DG, Forbes GS, Thielen K, Nichols D, O'Fallon WM, et al: Unruptured intracranial aneurysms: Natural history, clinical outcome, and risks of surgical and endovascular treatment. Lancet 362: 103-110, 2003.

2. Choi IS and David C: Giant intracranial aneurysms: Development, clinical presentation and treatment. Eur J Radiol 46: 178-194, 2003

3. Lawton MT and Spetzler RF: Surgical strategies for giant intracranial aneurysms. Neurosurg Clin N Am 9: 725-742, 1998.

4. Chen J, Zhu J, He J, Wang Y, Chen L, Zhang C, Zhou J and Yang L: Ultra-early microsurgical treatment within $24 \mathrm{~h}$ of SAH improves prognosis of poor-grade aneurysm combined with intracerebral hematoma. Oncol Lett 11: 3173-3178, 2016.

5. Hudgins RJ, Day AL, Quisling RG, Rhoton AL Jr, Sypert GW and Garcia-Bengochea F: Aneurysms of the posterior inferior cerebellar artery: A clinical and anatomical analysis. J Neurosurg 58: 381-387, 1983.

6. Locksley HB, Sahs AL and Sandler R: Report on the cooperative study of intracranial aneurysms and subarachnoid hemorrhage: Part 3-subarachnoid hemorrhage unrelated to intracranial aneurysm and A-V malformation: A study of associated diseases and prognosis. J Neurosurg 24: 1034-1056, 1966.

7. Maimon S, Saraf-Lavi E, Rappaport ZH and Bachar G: Endovascular treatment of isolated dissecting aneurysm of the posterior inferior cerebellar artery. AJNR Am J Neuroradiol 27: 527-532, 2006.

8. Wetjen NM, Link MJ, Reimer R, Nichols DA and Giannini C: Clinical presentation and surgical management of dissecting posterior inferior cerebellar artery aneurysms: 2 case reports. Surg Neurol 64: 462-467, 2005.

9. Ishikawa T, Suzuki A and Yasui N: Distal posterior inferior cerebellar aneurysms: Report of 12 cases. Neurol Med Chir (Tokyo) 30: 100-108, 1990.

10. Al-khayat H, Al-Khayat H, Beshay J, Manner D and White J: Vertebral artery-posteroinferior cerebellar artery aneurysms: Clinical and lower cranial nerve outcomes in 52 patients. Neurosurgery 56: 2-11, 2005.

11. Lewis SB, Chang DJ, Peace DA, Lafrentz PJ and Day AL: Distal posterior inferior cerebellar artery aneurysms: Clinical features and management. J Neurosurg 97: 756-766, 2002.

12. Yasargil MG: Vertebrobasilar aneurysms, in microneurosurgery: Clinical considerations, surgery of the intracranial aneurysms and results. Stuttgart, Georg Thieme 2: 232-295, 1984.

13. Sedat J, Chau Y, Mahagne MH, Bourg V, Lonjon M and Paquis P: Dissection of the posteroinferior cerebellar artery: Clinical characteristics and long-term follow-up in five cases. Cerebrovasc Dis 24: 183-190, 2007.

14. Hamada J, Nagahiro S, Mimata C, Kaku T and Ushio Y: Reconstruction of the posterior inferior cerebellar artery in the treatment of giant aneurysms: Report of two cases. J Neurosurg 85: 496-499, 1996.

15. Yamakawa H, Kaku Y, Yoshimura S, Ohkuma A and Sakai N: Two cases of dissecting aneurysm of the distal posterior inferior cerebellar artery: Possible involvement of segmental mediolytic arteriopathy in the pathogenesis. Clin Neurol Neurosurg 107: 117-122, 2005.

16. Horowitz M, Kopitnik T, Landreneau F, Krummerman J, Batjer HH, Thomas G and Samson D: Posteroinferior cerebellar artery aneurysms: Surgical results for 38 patients. Neurosurgery 43: 1026-1032, 1998. 
17. Wu J, Xu F, Yu ZQ, Zhou YX, Cui G, Li XD, Zhou D, Zhang SM and Wang $Z$ : Clinical experiences of ruptured posteroinferior cerebellar artery aneurysms and anatomical analysis in the Cadaver in a single center of China. Clinical Neurology and Neurosurgery 114: 366-371, 2012.

18. Mukonoweshuro W, Laitt RD and Hughes DG: Endovascular treatment of PICA aneurysms. Neuroradiology 45: 188-192, 2003.

19. Ogilvy CS, Hoh BL, Singer RJ and Putman CM: Clinical and radiographic outcome in the management of posterior circulation aneurysms by use of direct surgical or endovascular techniques. Neurosurgery 51: 14-21; discussion 21-22, 2002.

20. Byrne JV, Sohn MJ, Molyneux AJ and Chir B: Five-year experience in using coil embolization for ruptured intracranial aneurysms: Outcomes and incidence of late rebleeding. J Neurosurg 90: 656-663, 1999.

21. Horiuchi T, Tanaka Y, Hongo K, Nitta J, Kusano Y and Kobayashi S: Characteristics of distal posteroinferior cerebellar artery aneurysms. Neurosurgery 53: 589-596, 2003.
22. Macchi V, Porzionato A, Parenti A and De Caro R: The course of the posterior inferior cerebellar artery may be related to its level of origin. Surg Radiol Anat 26: 60-65, 2004.

23. Lemole GM Jr, Henn J, Javedan S, Deshmukh V and Spetzler RF: Cerebral revascularization performed using posterior inferior cerebellar artery-posterior inferior cerebellar artery bypass: Report of four cases and literature review. J Neurosurg 97: 219-223, 2002

24. Yoon SM, Shim JJ, Kim SH and Chang JC: Bilateral vertebral artery dissecting aneurysms presenting with subarachnoid hemorrhage treated by staged coil trapping and covered stents graft. J Korean Neurosurg Soc 51: 155-159, 2012.

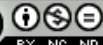

This work is licensed under a Creative Commons Attribution-NonCommercial-NoDerivatives 4.0 International (CC BY-NC-ND 4.0) License. 